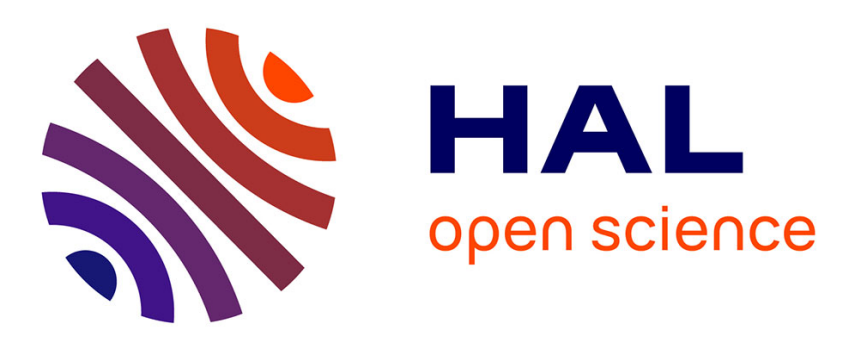

\title{
Pressure-induced spin-crossover features at variable temperature revealed by in situ synchrotron powder $\mathrm{x}$-ray diffraction
}

Élodie Tailleur, Mathieu Marchivie, Jean-Paul Itié, Patrick Rosa, Nathalie

Daro, Phillippe Guionneau

\section{To cite this version:}

Élodie Tailleur, Mathieu Marchivie, Jean-Paul Itié, Patrick Rosa, Nathalie Daro, et al.. Pressureinduced spin-crossover features at variable temperature revealed by in situ synchrotron powder x-ray diffraction. Chemistry - A European Journal, 2018, 24 (54), pp.14495-14499. 10.1002/chem.201802828. hal-01880970

\section{HAL Id: hal-01880970 \\ https://hal.science/hal-01880970}

Submitted on 25 Sep 2018

HAL is a multi-disciplinary open access archive for the deposit and dissemination of scientific research documents, whether they are published or not. The documents may come from teaching and research institutions in France or abroad, or from public or private research centers.
L'archive ouverte pluridisciplinaire HAL, est destinée au dépôt et à la diffusion de documents scientifiques de niveau recherche, publiés ou non, émanant des établissements d'enseignement et de recherche français ou étrangers, des laboratoires publics ou privés. 


\title{
Pressure-induced spin-crossover features at variable temperature revealed by in situ synchrotron powder X-ray diffraction
}

\author{
Tailleur E. ${ }^{1}$, Marchivie M. ${ }^{1}$, Itié J.-P. ${ }^{2}$, Rosa P. ${ }^{1}$, Daro N. ${ }^{1}$, Guionneau Ph. ${ }^{1}$ \\ ${ }^{1}$ CNRS, Univ. Bordeaux, ICMCB, UMR 5026, F-33600 Pessac, France \\ ${ }^{2}$ Synchrotron SOLEIL, L'Orme des Merisier, Saint-Aubin, BP 49, 91192 Gif-sur-Yvette, France
}

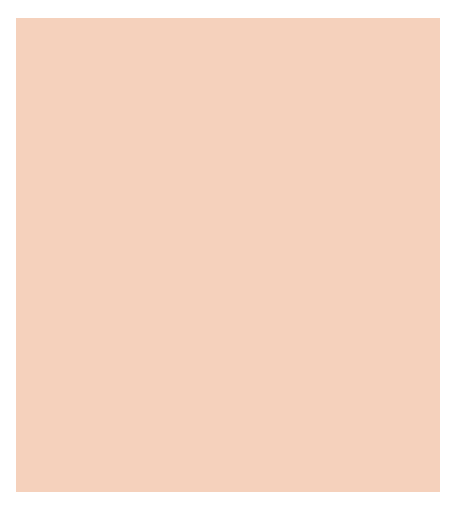

\begin{abstract}
An accurate high-pressure $\mathrm{X}$-ray diffraction investigation, at various temperatures, on a powder of a spin-crossover (SCO) complex has allowed the rare deconvolution of the structural features of the high-spin and low-spin phases. As a result, the pressure dependence of the structural parameters of the highspin and low-spin phases can be discussed independently in the pressure domain where both phases co-exist within the powder. Consequently, crucial unprecedented information is given, such as the variation of bulk moduli with temperature, similar here in amplitude for both spin phases, the temperature-dependence of the pressure-induced SCO abruptness, the temperature dependence of the pressure at which SCO occurs, and arguments for a possible piezohysteresis. Performed on the molecular complex [Fe(PM$\left.\mathrm{PeA})_{2}(\mathrm{NCSe})_{2}\right] \quad(\mathrm{PM}-\mathrm{PeA}=\mathrm{N}-(2$ - -pyridylmethylene $)-4-$ (phenylethynyl) aniline), this study reveals a pressureinduced SCO at $0.16 \mathrm{GPa}$ and demonstrates that, when increasing temperature, the pressure of transition increases linearly, the abruptness of the pressure-induced SCO strongly increases, and the bulk moduli decrease.
\end{abstract}




\section{Introduction}

The spin-crossover phenomenon (SCO) corresponds to a change in the electronic configuration from a high spin (HS) state to a low spin (LS) state, or conversely, mostly occurring in $3 \mathrm{~d}$ transition metals with electronic configurations $\mathrm{d}^{4}$ to $\mathrm{d}^{7}$, manifesting as a switch of optical, magnetic, and structural properties. Known stimuli are temperature, light irradiation, pressure, electric current and field.1 The relation between the SCO features and the way temperature and light irradiation are applied appears relatively well known, thanks to thorough and numerous investigations, even though many aspects are still discussed.1, 2 On the other hand, in spite of pressure being identified a long time ago as a key thermodynamic factor in spin-crossover, technical difficulties have made experimental high-pressure investigations uncommon. In this context, the investigation of SCO solids combining high-pressure (HP) and variable temperature (VT) are even scarcer. High-pressure X-ray structural analyses on powders are uncommon, since the small sample volume available in a diamond anvil cell (DAC) requires highflux beams. The present paper represents one of the very rare X-ray diffraction studies that explore a SCO material in powder form while varying pressure and temperature conditions, in order to investigate the effect of temperature on the pressure-induced SCO, including unit cell parameters, bulk moduli, and abruptness of transition. The use of pressure to perturb the electronic and physical structure of coordination compounds is stimulating, since it can lead to unknown phase transitions with new properties and therefore it opens potentially a variety of applications, including piezochromism.3-6 Nevertheless, regarding the effects of pressure on SCO solids, numerous fundamental aspects are still strongly debated or almost unknown.1, 2, 7-9 In octahedral iron(II) complexes, SCO occurs as the electronic configuration of the iron switches from a paramagnetic HS $\left(S=2, \mathrm{t}_{2 \mathrm{~g}}{ }^{4} \mathrm{eg}^{2}\right)$ to a diamagnetic $\operatorname{LS}\left(S=0, \mathrm{t}_{2 \mathrm{~g}}{ }^{6} \mathrm{eg}_{\mathrm{g}}{ }^{0}\right)$ state. Consequently, for a given molecular complex, the LS phase usually corresponds to a lower volume than the HS phase due to the occupation by electrons of anti-bonding molecular orbitals in the HS state that increases the iron(II)-ligand bond lengths. As a result, applying a mechanical pressure on a material that is in the HS state, favors the occurrence of SCO.7 The effects of the concomitant variations of pressure and temperature on SCO solids are much less clear. The general expectation based on thermodynamics and/or theoretical considerations is that with increasing pressure, the SCO temperature increases, the SCO becomes more gradual, and the LS residue increases.9-14 Whereas many of the experiments made in the last four decades support these expectations, some counter-examples were also reported showing the complexity of SCO materials.7, 15-17 In situ diffraction investigations under pressure are relatively scarce despite being presently considered as one of the major challenges in the SCO community. Since molecular single-crystals can be rapidly damaged upon application of pressure, powder X-ray diffraction (PXRD) can appear more appropriate for in situ investigations under pressure. Moreover, PXRD also potentially brings data at the microstructural scale, an aspect that is, to date, almost unexplored in SCO materials under pressure. In this article, the high-pressure study is focused on one of the very rare compounds showing a thermal SCO truly centered on room-temperature and reported recently:18 [Fe(PM-PeA $)_{2}(\mathrm{NCSe})_{2}$ ] [with PM-PeA $=N$-(2'-pyridylmethylene)-4-(phenylethynyl) aniline] presents a temperature induced SCO centered at $286 \mathrm{~K}$ with a hysteresis width of $41 \mathrm{~K}$. SCO occurs in this compound, together with a structural transition corresponding to an increase of symmetry at low temperature, from a HS monoclinic $P 2_{1} / c$ phase to a LS orthorhombic $P c c n$ phase. This rather unusual transition promises a priori a noteworthy behavior under pressure at variable temperature. Single-crystals are damaged upon SCO, which makes powder investigations preferable. This study aims to probe in situ the SCO phenomenon by PXRD combining high pressure and temperature. The high-pressure results at room temperature are presented first, followed by the pressure-temperature study.

\section{Results and Discussion}

A series of forty-four X-ray patterns have been obtained at room temperature after integration of the X-ray diffraction images (see Supporting Information) recorded in the pressure range 0.04-0.92 GPa. Three conclusions 
can be directly drawn. Firstly, the $\left[\mathrm{Fe}(\mathrm{PM}-\mathrm{PeA})_{2}(\mathrm{NCSe})_{2}\right]$ compound undergoes a pressure-induced SCO that is well evidenced by the decrease of the intensity of the Bragg peaks corresponding to the HS state, and the increase of the intensity of the Bragg peaks corresponding to the LS state. This evolution is clearly discernible on the two characteristic (110) Bragg peaks of the HS and LS phases (Figure 1). Secondly, the pressure-induced SCO is associated to the same structural transition as the one observed for the isobaric variable temperature-induced SCO (Figure 2 and Supporting Information). Thirdly, the coexistence of HS and LS phase is a proof of a first order transition, with a nucleation and domain growth phenomenon.19 The data are of sufficient quality to run Rietveld refinements at all the investigated pressures, notably leading to reliable information on the pressure-dependence of the unit cell parameters or volume, and of the HS/LS phase ratio as discussed below. The intensity of the Bragg peaks gives the percentage of HS phase within the powder sample, which offers direct observation of the pressure-induced SCO (Figures 1 and 3). The fine tuning of pressure with a data point every 0.02 GPa allows a very accurate track of the pressure-induced SCO. The pressure of transition, noted $P_{1 / 2}$, is defined here as the pressure where the ratio HS/LS is $50 \%$, by analogy to $T_{1 / 2}$ commonly used for the thermally induced SCO. $P_{1 / 2} \uparrow$ or $P_{1 / 2} \downarrow$ will be further used to account for the $P_{1 / 2}$ corresponding to an increase or decrease of pressure, respectively. At room temperature, $P_{1 / 2} \uparrow$ is $0.16 \mathrm{GPa}$ for the investigated compound.

When working on powders, it is possible-although rarely done - to perform an independent refinement of unit cell parameters and volume of both HS and LS phases (see Supporting Information). In the present case, remarkably, it has been possible to determine in parallel the HS phase structural parameters in its whole pressure range of presence, $0-0.75 \mathrm{GPa}$, and the LS structural parameters over 0.06-0.92 GPa. Consequently, unlike in single-crystal experiments where the unit cell parameters and volume obtained at one given pressure is an average of the HS and LS values within the sample, or corresponds to the sole major phase, here, it is possible to extract independently the HS and LS unit cell parameters and volumes. To the best of our knowledge, it is the first time that such a result is achieved. It offers a clear description of the pressure-induced SCO and a valuable description of the HS and LS phases over the whole pressure range as shown in Figure 2. Note however that the HS phase fraction appears very small in the range $0.36-0.75 \mathrm{GPa}$, leading to poorly reliable refinements and therefore, the HS phase study below focuses on the range of significant presence, that is, 0-0.36 GPa.

In this compound the LS phase is clearly observed early in the experiment, whereas the HS phase, as said above, is also observed over a large pressure range. Consequently, for this compound, the domain of bi-stability appears large. The variation of the unit cell volumes truly reflects here the pressure dependence of the HS and LS phase volumes, which offers a rare opportunity to extract the bulk modulus, a crucial but rare data, for each phase in a separate mode. Bulk moduli have been already obtained experimentally for some SCO materials, but their meaningfulness was subject to discussion since they were based on data corresponding to the average volume for two phases.16, 20-22 This is not the case here and, probably for the first time, the bulk modulus of each phase can be obtained based on data within the same pressure range.

The bulk modulus, defined as $K=-V \frac{\partial P}{\partial V}$, represents the inverse of the compressibility of a solid and measures its resistance to compression. The lower the bulk modulus, the higher the compressibility and the more easily deformable is the sample. Bulk moduli for the HS phase and the LS phase, are 6.1 and $5.4 \mathrm{GPa}$, respectively. These values have been obtained by fitting volume versus pressure data with a third order Birch-Murnaghan equation of state, using EOS-FIT.23, 24 These values are comparable to the one reported in literature for molecular materials.21 The strength of the present experiment is that the variation of the HS and LS volumes as a function of pressure are determined independently, and consequently the equation of state given by the fit has a true physical meaning compared to the ones reported in the literature so far, where the pressure dependence volume involved both contraction and volume changes due to the SCO.2, 21 This result is a real step forward into the analysis of the SCO phenomenon. Here, the closeness of bulk moduli for the HS and LS phases reveals that the HS and LS phases have a similar compressibility. This looks coherent, since even if the molecular volume is lower in the LS state leading to a higher density, the intermolecular interactions are rather similar in the HS and LS states and differ only according to very fine features. It demonstrates, experimentally, that in this compound the bulk modulus is not a crucial parameter to differentiate the HS and LS phases. It is worth remembering that the bulk 
modulus only stands for an isotropic compression, whereas in molecular materials the high-pressure modifications can strongly depend on the crystallographic directions. Additionally, the SCO phenomenon is known to act in a highly anisotropic mode on the material.2, 20 In the present compound, in the HS phase, the $c$ axis decreases by $10 \%$ whereas the $a$ and $b$ axes remain almost stable with a compression of 2.0 and $1.8 \%$ respectively. Thus, the compression of the unit cell in the HS state is highly anisotropic, independently from the SCO. In the LS phase, the compression appears less anisotropic. The $b$ axis is the most compressible since it decreases by $5.3 \%$ when the $a$ and $c$ axes decrease, respectively by 2.0 and $3.8 \%$. Consequently, a close examination taking into account anisotropy enhances significant differences in the pressure behaviors of the HS and LS phases. These variations can be related to the crystal packing and therefore to the feature of the intermolecular interaction topology. Within the crystal-packing of the title compound, the main directions of compression correspond indeed to directions where there is a relative free space between molecules, that is, where intermolecular interactions appear weak. For example, the well-known $\mathrm{H}-\mathrm{H}$ interaction that is a typical feature of this family of compounds, and that is directed along the $b$ axis, is known to disappear in the LS state.18 Furthermore, in order to construct the $(P, T)$ diagram and to investigate the temperature dependence of the pressure-induced SCO in this compound, similar experiments and analyses as above have been performed at three higher temperatures: 313, 333 and 353 K. A fresh powder, fully HS, has been used for each temperature studied. The SCO curves deduced from the HS ratio were determined at each temperature as shown in Figure 3. The temperature dependence of the SCO pressure transition, $P_{1 / 2} \uparrow$, has been obtained (Figure 4). Several conclusions can be extracted from those experiments. First, $P_{1 / 2} \uparrow$ increases when the temperature increases. This seems to be consistent because high temperature stabilizes the HS state. Moreover, as shown in Figure 4 , the $P$ versus $T$ relation is linear, here with a slope of $2.21(25) \times 10^{-3} \mathrm{GPa} \mathrm{K}^{-1}$. This value, although slightly lower, is of the same order of magnitude as those reported in literature, which are between 5 and $8 \times 10^{-3}$ GPa K $\mathrm{K}^{-1} .22,25,26$ This was expected from theory,13, 20 but the present in situ investigation offers one of the rare experimental evidences of the pressure-temperature linear relationship in SCO solids. Such nice correlation has been obtained essentially because experiments were performed at a fixed temperature and variable pressure, in contrast to what is usually done, leading here to very reliable $P / T$ data. Most of all, the independent refinements of the HS and LS phases result in a meaningful pressure $P_{1 / 2} \uparrow$ value. Then, looking at the HS fraction curves at various temperatures (Figure 3), it appears clearly that the abruptness of the SCO increases with temperature. To better characterize this abruptness, the $\Delta P_{60}$ parameter, which is the difference between $P_{80}$ and $P_{20}$, pressures corresponding to the ones at which the compound is $80 \%$ HS and $20 \%$ HS respectively is shown in Table $\underline{1}$. Therefore, the smaller $\Delta P_{60}$, the greater is the abruptness. Here, clearly, the abruptness of the pressure-induced SCO increases at high temperature, even if it is not the case for $313 \mathrm{~K}$ with a $\Delta P_{60}$ very close to the one at room temperature, but significantly higher than those at 333 and $353 \mathrm{~K}$. Furthermore, the bulk modulus of both phases decreases continuously with temperature (Table 1) indicating that the thermal dilation makes the material more compressible. Detailed dependences of the unit cell parameters with pressure are given in the Supporting Information.

The reversibility of the HS to LS switch was studied by decreasing the pressure. In all cases the compound remains LS until 0.1 GPa, then the LS to HS switch starts and leads at ambient pressure to an HS fraction of about $12 \%$ for the room-temperature, and $50 \%$ for the 313, 333 and $353 \mathrm{~K}$ experiments (Supporting Information). A negative pressure should probably be applied to recover the full HS phase. This partial reversal of the compound to the HS state can be explained either by the existence of a piezo-hysteresis in this compound, or by some irreversible evolution of the compound resulting in a fixed HS fraction. It would of course be desirable to check the compound properties after release of the pressure through magnetic measurements on the powder.

Unfortunately, the impossibility of recovering such a small amount from the silicon oil used as pressure medium, combined with the known problems of performing fixed pressure-variable temperature SQUID measurements25 on significant amounts, thus requiring a piston-cell and preventing the use of better performing DAC setups,26 made such a check impossible. Otherwise we stress that no significant changes are observed in the diffractogram 
measured after releasing the pressure (Supporting Information), which casts into doubt the formation of a new phase with a different behavior, especially considering the very moderate pressures applied. The formation of a blocked phase resulting from pressure should lead to a more or less constant LS fraction, whereas the latter is seen to depend on the measurement temperature. These arguments thus support the hypothesis that a piezo-hysteresis is likely observed in this compound. This hysteresis can be characterized by $\Delta P$ as estimated in Table 1 . In this frame the increase of the HS fraction after pressure release for the high temperature experiments corresponds thus to higher pressures of transition. This is counterintuitive since it would then support the broadening of the piezohysteresis with increasing temperature. By extension, this behavior must correspond to wider thermal-hysteresis with increasing pressure. However, this behavior has been observed several times and has been attributed either to the appearance of a phase transition, or to a strong anisotropic variation of the unit cell, and therefore to a large variation of the bulk modulus with pressure.15, 27-29 Interestingly, here, $K^{\prime}$ that corresponds to the derivation of $K$ with pressure is 7 for the HS phase, comparable to the only other $K^{\prime}$ value reported in the literature,21 and 14 for LS phase, which is twice as high. Consequently, the present results seem to provide one of the rare experimental evidences for the broadening of the hysteresis with increasing pressure.

\section{Conclusion}

To sum up, the $\mathrm{SCO}\left[\mathrm{Fe}(\mathrm{PM}-\mathrm{PeA})_{2}(\mathrm{NCSe})_{2}\right]$ compound presents an unprecedented behavior under pressure, with a transition at a relatively low pressure $(0.16 \mathrm{GPa})$ at room temperature, together with strong presumptions of a very large piezo-hysteresis. The present study also gives an experimental proof of the linear evolution of the pressure of transition with increasing temperature. Moreover, novel aspects are accessible here, such as the modifications of the abruptness of the pressure-induced SCO and the bulk moduli as a function of temperature, thanks to the possibility to determine independently the behaviors of the HS and LS phases in the large pressure range of coexistence. In situ PXRD under pressure, as used here, is pioneering in the field of SCO materials and many of the points investigated here could be extended to other material fields - to name just one aspect, the determination of bulk modulus of concomitant phases might be of interest within any field involving phase transitions.

\section{Experimental section}

The experiments have been performed on the PSICHE beamline at the SOLEIL synchrotron. A membrane diamond anvil cell allows a very fine tuning of the pressure on the sample. The gasket is made of Cu-Be soft alloy, well adapted for the low pressure working range, and silicone oil is used as pressure transmitter.30, 31 The pressure was measured in situ using the position of the (200) and (220) diffraction peaks and the equation of state of $\mathrm{NaCl} 32$ giving a precision close to $0.01 \mathrm{GPa}$. Experiments have been conducted at fixed temperature and variable pressure. In this way, there is only one variable and the $P / T$ couple is perfectly controlled. Around fifty different pressures were recorded at 293, 313, 333 and $353 \mathrm{~K}$. The very low instrumental broadening of the synchrotron radiation was a key point to properly refine the different phase parameters and fractions when mixtures occur, which was the main goal of the present study. Detailed experimental and data-analysis features are in the Supporting Information, including a full list of $R$ and $R_{\mathrm{wp}}$ factors. Note that this work corresponds to the achievements of about 180 Rietveld refinements. In all cases, a Le Bail refinement was performed prior to the Rietveld refinement.

\section{Acknowledgements}

The authors gratefully acknowledge the Agence Nationale de la Recherche for the financial support under the grant ANR FemtoMat 13-BS046002, the Region Nouvelle Aquitaine. The University of Bordeaux has provided the PhD grant for E.T. 


\section{References}

1. M. A. Halcrow, Spin-Crossover Materials: Properties and Applications, Wiley-Blackwell, Oxford, 2013.

2. P. Guionneau, Dalton Trans. 2014, 43, 382-393.

3. J.-F. Létard, P. Guionneau, L. Goux-Capes, in Spin Crossover in Transition Metal Compounds III (Eds.: P. Gütlich, H. A. Goodwin), Springer, Heidelberg, 2004, pp. 221-249.

4. K. Boukheddaden, M. H. Ritti, G. Bouchez, M. Sy, M. M. Dîrtu, M. Parlier, J. Linares, Y. Garcia, J. Phys. Chem. C 2018, 122, 7597-7604.

5. J. Linares, E. Codjovi, Y. Garcia, Sensors 2012, 12, 4479-4492.

6. H. Li, H. Peng, Curr. Opin. Colloid Interface Sci. 2018, 35, 9-16.

7. G. G. Levchenko, A. V. Khristov, V. N. Varyukhin, Low Temp. Phys. 2014, 40, 571-585.

8. P. Gütlich, A. B. Gaspar, Y. Garcia, Beilstein J. Org. Chem. 2013, 9, 342-391.

9. A. Bousseksou, G. Molnár, L. Salmon, W. Nicolazzi, Chem. Soc. Rev. 2011, 40, 3313-3335.

10. P. Gütlich, A. B. Gaspar, V. Ksenofontov, Y. Garcia, J. Phys. Condens. Matter 2004, 16, S1087-S1108.

11. S. Bonhommeau, G. Molnár, M. Goiran, K. Boukheddaden, A. Bousseksou, Phys. Rev. B 2006, $74,064424$.

12. C. Roux, J. Zarembowitch, J.-P. Itié, A. Polian, M. Verdaguer, Inorg. Chem. 1996, 35, 574-580.

13. C. P. Slichter, H. G. Drickamer, J. Chem. Phys. 1972, 56, 2142-2160.

14. L. Stoleriu, P. Chakraborty, A. Hauser, A. Stancu, C. Enachescu, Phys. Rev. B 2011, 84, 134102.

15. V. Ksenofontov, G. Levchenko, H. Spiering, P. Gutlich, J. F. Letard, Y. Bouhedja, O. Kahn, Chem. Phys. Lett. 1998, 294, 545-553.

16. H. J. Shepherd, T. Palamarciuc, P. Rosa, P. Guionneau, G. Molnár, J.-F. Létard, A. Bousseksou, Angew. Chem. Int. Ed. 2012, 51, 3910-3914; Angew. Chem. 2012, 124, 3976-3980.

17. N. Paradis, F. Le Gac, P. Guionneau, A. Largeteau, D. Yufit, P. Rosa, J.-F. Létard, G. Chastanet, Magnetochemistry 2016, 2, 15.

18. E. Tailleur, M. Marchivie, N. Daro, G. Chastanet, P. Guionneau, Chem. Commun. 2017, 53, 4763-4766.

19. M. Buron-Le Cointe, J. Hébert, C. Baldé, N. Moisan, L. Toupet, P. Guionneau, J. F. Létard, E. Freysz, H. Cailleau, E. Collet, Phys. Rev. B 2012, 85, 064114.

20. P. Guionneau, M. Marchivie, G. Bravic, J.-F. Létard, D. Chasseau, Top. Curr. Chem. 2004, 234, 97-128.

21. H. J. Shepherd, P. Rosa, L. Vendier, N. Casati, J.-F. Létard, A. Bousseksou, P. Guionneau, G. Molnár, Phys. Chem. Chem. Phys. 2012, 14, 5265-5271.

22. H. J. Shepherd, S. Bonnet, P. Guionneau, S. Bedoui, G. Garbarino, W. Nicolazzi, A. Bousseksou, G. Molnár, Phys. Rev. B 2011, 84, 144107.

23. J. Gonzalez-Platas, M. Alvaro, F. Nestola, R. Angel, J. Appl. Crystallogr. 2016, 49, 1377-1382.

24. R. J. Angel, M. Alvaro, J. Gonzalez-Platas, Z. Kristallogr. 2014, 229, 405-419.

25. J. Sanchez-Benitez, S. Tancharakorn, M. K. Hutchison, K. V. Kamenev, J. Phys.: Conf. Ser. 2008, $121,122001$.

26. G. Giriat, W. Wang, J. P. Attfield, A. D. Huxley, K. V. Kamenev, Rev. Sci. Instrum. 2010, 81, 073905.

27. H. Spiering, N. Willenbacher, J. Phys. Condens. Matter 1989, 1, 10089-10105.

28. V. Ksenofontov, H. Spiering, A. Schreiner, G. Levchenko, H. Goodwin, P. Gütlich, J. Phys. Chem. Solids 1999, 60, 393-399.

29. P. Gütlich, V. Ksenofontov, A. B. Gaspar, Coord. Chem. Rev. 2005, 249, 1811-1829.

30. L. Merrill, W. A. Bassett, Rev. Sci. Instrum. 1974, 45, 290-294.

31. Y. Shen, R. S. Kumar, M. Pravica, M. F. Nicol, Rev. Sci. Instrum. 2004, 75, 4450-4454.

32. P. I. Dorogokupets, A. Dewaele, High Press. Res. 2007, 27, 431-446. 


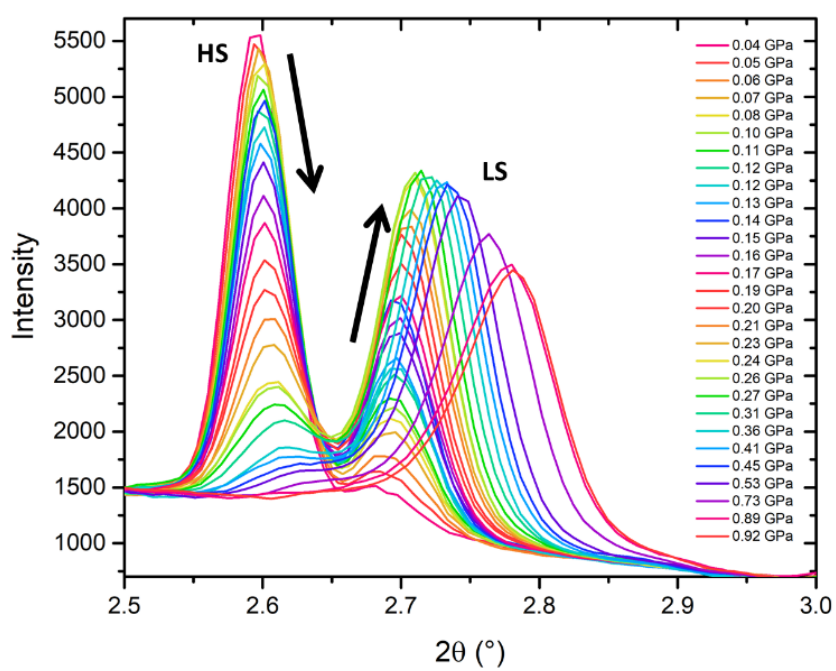

Figure 1. Evolution of the intensity of the (110) Bragg peaks of the HS phase (peak at $2 \theta \sim 2.6^{\circ}$ ) and LS phase (peak at $2 \theta>2.7^{\circ}$ ) with increasing pressure at room temperature.

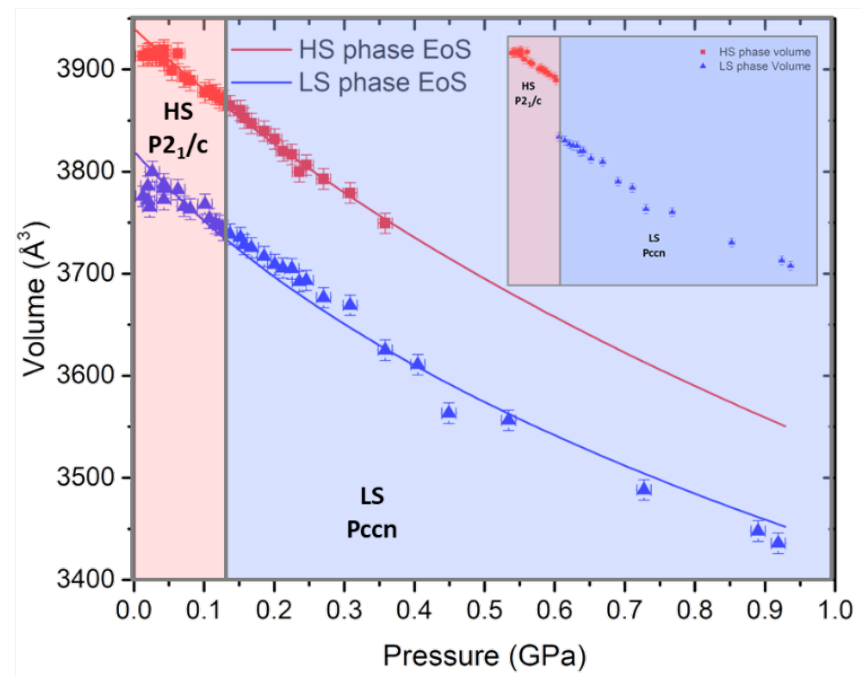

Figure 2. Innovative representation of the pressure dependence unit-cell volume (red: HS; blue: LS) obtained from the independent but concomitant refinements of both the HS and LS phases in contrast with the classical view (insert). Curves represent the equation of state calculated with a third order Birch-Murnaghan model 

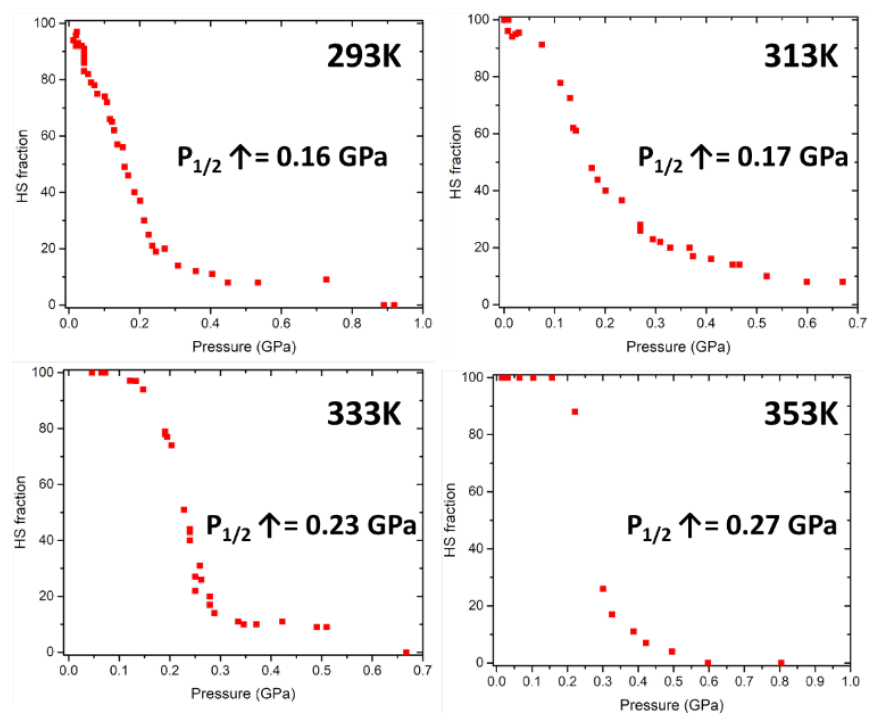

Figure 3. Pressure dependence of the HS fraction deduced from the Rietveld refinements X-ray diffraction patterns recorded in situ under pressure at four temperatures $293 \mathrm{~K}, 313 \mathrm{~K}, 333 \mathrm{~K}$ and $353 \mathrm{~K}$. Standard deviations on $\mathrm{P}_{1 / 2}$ are estimated to $0.02 \mathrm{GPa}$.

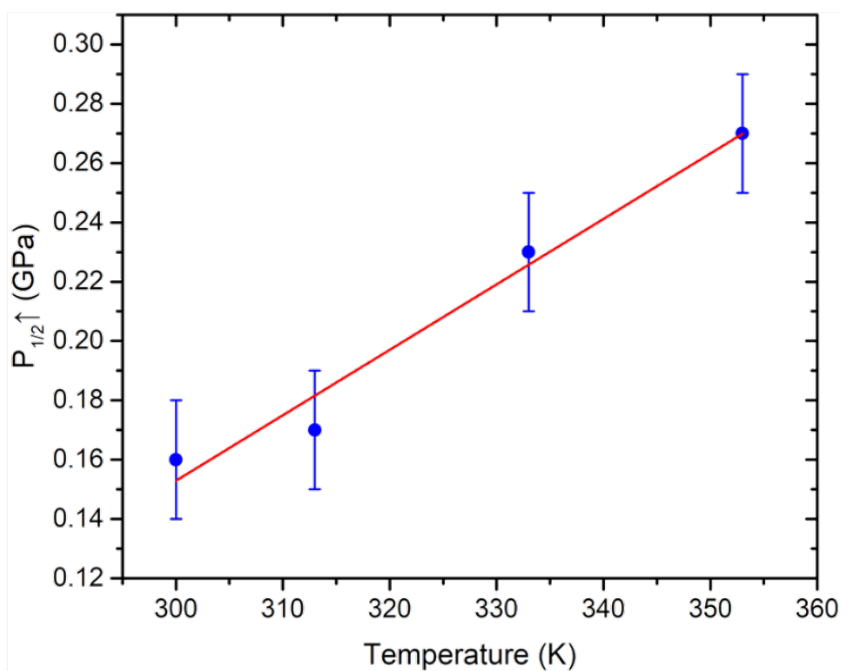

Figure 4. Variation of the pressure of SCO observed by increasing the pressure at a fixed temperature, $\mathrm{P}_{1 / 2} \uparrow$, as a function of the temperature. The values of pressure are calculated from powder X-ray diffraction data Rietveld refinements of the HS and LS phases leading to the HS and LS fractions. $\mathrm{P}_{1 / 2} \uparrow$ corresponds to the pressure where HS and LS phases count for $50 \%$ each. 
Table 1. Features derived from the high-pressure variable PXRD investigations from the pressure-induced HS ratio curves. Characteristic pressures $\mathrm{P}_{80}, \mathrm{P}_{20}, \Delta \mathrm{P}_{60}$, bulk moduli for the $\mathrm{HS}\left(\mathrm{K}_{\mathrm{HS}}\right)$ and $\mathrm{LS}\left(\mathrm{K}_{\mathrm{LS}}\right)$ phases and characteristic pressure transition and piezo-hysteresis. See text for definitions.

\begin{tabular}{lllll}
\hline & $293 \mathrm{~K}$ & $313 \mathrm{~K}$ & $333 \mathrm{~K}$ & $353 \mathrm{~K}$ \\
\hline $\mathrm{P}_{80}(\mathrm{GPa})$ & 0.059 & 0.104 & 0.189 & 0.231 \\
$\mathrm{P}_{20}(\mathrm{GPa})$ & 0.241 & 0.329 & 0.281 & 0.318 \\
$\Delta \mathrm{P}_{60}(\mathrm{GPa})$ & 0.182 & 0.225 & 0.092 & 0.087 \\
$\mathrm{~K}_{\mathrm{HS}}(\mathrm{GPa})$ & $6.2(2)$ & $5.1(1)$ & $4.6(1)$ & $4.4(1)$ \\
$\mathrm{K}_{\mathrm{LS}}(\mathrm{GPa})$ & $4.9(1)$ & $4.5(1)$ & $3.9(1)$ & $3.6(2)$ \\
$\mathrm{P}_{1 / 2} \uparrow(\mathrm{GPa})$ & 0.16 & 0.17 & 0.23 & 0.27 \\
$\mathrm{P}_{1 / 2} \downarrow(\mathrm{GPa})$ & $<0$ & 0.0 & 0.0 & 0.0 \\
$\Delta \mathrm{P}(\mathrm{GPa})$ & $>0.16$ & 0.17 & 0.23 & 0.27 \\
\hline
\end{tabular}

[a] Characteristic pressures $P_{80}, P_{20}, \Delta P_{60}$, bulk modulus for the HS $\left(K_{\mathrm{HS}}\right)$ and LS $\left(K_{\mathrm{LS}}\right)$ phases and characteristic pressure transition and piezo-hysteresis. See text for definitions. 